\title{
Towards a Narrative Mind: The Creation of Coherent Life Stories for Believable Virtual Agents
}

\author{
Wan Ching Ho and Kerstin Dautenhahn \\ Adaptive Systems Research Group, School of Computer Science \\ University of Hertfordshire, Hatfield, Hertfordshire, AL10 9AB, UK \\ \{w.c.ho, k.dautenhahn\}@herts.ac.uk
}

\begin{abstract}
This paper describes an approach to create coherent life stories for Intelligent Virtual Agents (IVAs) in order to achieve long-term believability. We integrate a computational autobiographic memory, which allows agents to remember significant past experiences and reconstruct their life stories from these experiences, into an emotion-driven planning architecture. Starting from the literature review on episodic memory modelling and narrative agents, we discuss design considerations for believable agents which interact with users repeatedly and over a long period of time. In the main body of the paper we present the narrative structure of human life stories. Based on this, we incorporate three essential discourse units and other characteristics into the design of the autobiographic memory structure. We outline part of the implementation of this memory architecture and describe the plan for evaluating the architecture in long-term user studies.
\end{abstract}

\section{Introduction}

In pursuing the ultimate goal of creating agents that give an "illusion of life" [1], Intelligent Virtual Agents (IVAs) researchers in the past decade have applied different mechanisms to enhance agents' believability. In addition to the efforts in advancing the computer graphics and language engines; from the perspective of cognitive architecture modelling, human personality and emotion models (e.g. BDI [2],OCC [3] and PSI $[4,5]$ ) have brought fruitful outcomes into this research direction. As a result, nowadays a large variety of software applications in education, entertainment and other areas are populated with believable agents for increasing the level of user engagement. So, what can still be missing?

We argue that the answer can be long-term believability. When considering the IVA's role as an one-off problem solving tool or a short-term interactive target, it is probably not necessary for the agent to inform human users what it remembers and learned from the past. There are circumstances, however, that require users to repeatedly interact with the same agent. For example, this includes a long-term companion agent which assists the user in various tasks on a day-to-day basis, or a conversational narrative agent which tells stories about 
itself to the user. Both types of agents are aiming at long-term and repeated interactions with a user. Thus, they shall require at least an extra cognitive component which commonly does not seem to exist in IVA applications - a long-term autobiographic memory. The reason for integrating this memory is obvious: A companion agent shall learn from the user through their interaction histories; similarly, a narrative agent shall not duplicate its story in order not to bore the user.

One may then argue that, in order to generate different stories, a narrative agent can select different bits of story content from a database with some predefined rules. This approach may be systematically feasible and the stories, with the high-level control on the narrative structure (e.g. defined in [6] and [7]), can make sense to us. Alternatively, when using intelligent characters and substantial involvement from users, emergent narrative $[8,9]$ creates flexible structures for real-time and interactive narrative with some constraints to the richness of the story contents. Both approaches, however, do not address the issue of long-term believability - while planning to unfold a story, the user's previous interactions and the character's own experiences in the story so far have not been addressed.

Focusing on the general aim in creating IVAs that appear believable and acceptable to humans, one way to facilitate this aim is allowing human users to have a better understanding of the behaviour expressed by agents. This direction, as indicated earlier by Sengers [10], requires agents to behave in a coherent ${ }^{1}$ manner so that the change from one behavior to another can be more understandable to human observers. In this paper one of our main goals is to model a human long-term memory computationally, thus we can attempt developing a sense of coherent "self" for IVAs.

In human beings, coherence is provided in the life stories of human beings [11] - since we communicate that sense of self and negotiate it with others through our life stories. Thus, we claim that the inclusion of it will be beneficial to believable agents, particularly in a narrative story-telling environment.

The rest of this paper is organised as follows: in the Related Work section, we first introduce the background literature in narrative storytelling agents, episodic memory modelling and our previous work in computational autobiographic memory. Next, we illustrate the implementation plan for long-term autobiographic memory with some preliminary results. We describe the on-going evaluation for the validation of our research hypothesis, namely that autobiographic memory can help agents improving their believability and user engagement through producing coherent life stories from the past experiences stored in their autobiographic memory. The Conclusion and Future Work sections conclude the paper.

\footnotetext{
${ }^{1}$ Sengers discussed a general problem of behavioral incoherence in IVA research - often human observers cannot understand why agents behave as they do; this phenomenon was described as 'schizophrenia' [10].
} 


\section{Related Work}

In Cognitive Science and Artificial Intelligence, the main stream of research in modelling human long-term memory and narrative tends to be associated with sense-making and problem-solving activities. "The human phenomenon" of rich and meaningful narrative, which can be found in all activities that involve the representation of events in time [12], has somewhat been neglected. In this section we introduce research in cognitive memory modelling and we present fundamental ideas of human/agent life stories as narrative. The design of longterm autobiographic memory for IVAs in the next section is influenced by and inspired from this literature.

\subsection{Modelling Long-term Memory}

To embodied computational agents that are capable of learning and adapting themselves to the environment, memory and representation are essential to be integrated in the architecture. Therefore, modelling a human-like long-term memory has always fascinated many AI researchers - and led to various memory models contributing significantly to the understanding of human cognition. For instance, Script, from Schank and Abelson [13], captures two important aspects of human memory in the perspective of developmental psychology: 1) it represents everyday events and activities, and 2) it has social-cultural components. Early models like Frame [14] and Script, however, represent knowledge in abstract static structures with little or no relation to "organic" developmental processes, or emotion.

In recent years, the use of temporal sequences of episodic events, in both robotic and virtual agents research, is a growing area. For example, by collecting relevant events stored in episodic memory, an explorative robot is able to reduce its state-estimate computation in the tasks of localising and building a cognitive map in a partially observable office environment [15]. Also, long-term episodic memory with attributing emotions may help a virtual robot to predict rewards from human users, thus facilitating human-robot interactions in a simple Peekaboo communication task [16].

Mirza et al. [17, 18] uses the concept of interaction histories, defined as 'the temporally extended, dynamically constructed and reconstructed, individual sensori-motor history of an agent situated and acting in its environment including the social environment'. This work is strongly inspired by dynamical systems approaches to memory and sensori-motor coordination. The approach does not lend itself naturally to be applied to virtual characters and believable virtual agents, since the memory content is not represented symbolically and it is thus not straightforward how to visualise and, more importantly, communicate it to human users.

The current research trend towards modelling a complete human episodic memory (e.g. episodic memory in Soar [19] and a generic episodic memory module [20]) establishes a common structure that consists of context, contents and 
outcomes/evaluation for agents to remember past experiences. These models were created to focus on the following three different aspects:

1. accuracy - how relevant situations can be retrieved from the memory.

2. scalability - how to accommodate a large number of episodes and not decrease significantly the performance of the system.

3. efficiency - how to optimise the storage and recall of memory contents

It is undoubted that these aspects are important for intelligent systems that are able to remember past events and anticipate future events. However, remembering past events and retrieving them in a timely manner, we argue, is not the only crucial part in creating IVAs interacting with human users - faster retrieval times cannot increase dramatically agents' believability. Likewise, highly accurate memory retrieval is not necessary in many situations involving believable agents that are meant to possess human-like memory: Human memory is not 'perfect' and can sometimes retrieve false memories or simply forget.

Brom et al. attempted to create a full episodic memory which stores more or less everything happening around the agent for the purpose of storytelling [21]. The authors claimed that the modelled episodic memory can answer specific questions from human users in real time regarding the agent's personal histories. Nevertheless, with the story scenario which was used in their paper, this memory was able to allow an agent to describe past actions in time but it failed to provide dramatic elements which facilitate an interesting piece of narrative. This may be due to the fact that the motivational and emotional states of the agents have not been fully integrated to determine the importance of a situation in an episode.

\subsection{Narrative Agents and Autobiographic Memory}

Bruner discussed in detail the narrative construction of human reality [22], in particular the social reality and the important role of stories in communication and social interaction. Following Bruner, Schank and Abelson suggested three cognitive features supported by the role of stories in individual and social understanding processes [23]:

1. Human knowledge is based on stories constructed around past experiences.

2. New experiences are interpreted in terms of old stories.

3. The content of story memories depends on whether and how they are told to others and these reconstituted memories form the basis of the individual's remembered self.

Based on these theoretical foundations, Dautenhahn proposed and discussed five different types of storytelling agents [24]:

1. Type 0: An agent that has a grandfather model of memory [23] - telling the same stories over and over again.

2. Type I: An agent that has a great variety of stories stored in its story-base. However each time the agent selects a single story randomly and tells it in exactly the same way as stored - not being situated to the conversational context. 
3. Type II: A narrative agent that selects the story to tell from its large story-base based on the current context of interaction. However, this agent does not listen to its interaction partner; thus it does not understand and incorporate other's stories in its own.

4. Type III: A believable narrative agent that is able to interpret the meaning and content of the story and finds in its own story-base the most similar story which is then adapted in order to produce an appropriate response.

5. Type IV: An autobiographic agent that can tell the most rich and interesting stories. This agent, like a living human being, links a story which is being told with its current sensory data, embodiment (e.g. using non-verbal behaviours), past experiences and personality.

The term autobiographic agents was first defined in[25, page 5] as "agents which are embodied and situated in a particular environment (including other agents), and which dynamically reconstruct their individual history (autobiography) during their lifetimes.". This individual history can help autobiographic agents to develop individualised social relationships and to communicate with others, which are characteristics of social intelligence. The idea of modelling autobiographic memory in agents was inspired from the human psychological phenomena regarding the assimilation of memory to autobiography and sharing it with others - which were suggested as a psychological approach to narrative:

- "...this social function of (autobiographic) memory underlines all of our storytelling, history-making narrative activities, and ultimately all of our accumulated knowledge systems." [26, page 12]

- Narrative is one of the most effective means of creating, maintaining and communicating the self [11].

A simple, but nevertheless first technical implementation of an autobiographic agent, using a Webots (Cyberbotics) simulation environment, was presented by Dautenhahn and Coles [27]. Here, an agent acted on the basis of its memories of past experiences and replayed a sequence of actions that previously led to a goal. Extending this work, Ho et al. developed and experimentally evaluated different types of computational memory architectures for Artificial Life autobiographic agents, e.g. in [28]. In attempting to solve the problem of behavioural incoherence from a user's perspective, we developed an Observer Interface that shows the dynamically changing contents of an agent's memory as well as stories received from other agents [29]. Thus, this interface was an attempt to allow the user to see coherence in the agents' behaviour, i.e. allowing them to understand why particular actions were taken. Thus, in this work the agents' behaviours can be understood as intentional, narratively structured and temporally grounded. Furthermore, the communication of experience can be seen to rely on emergent mixed narrative reconstructions combining the experiences of several agents, cf. details in [29]. 


\section{Narrative Autobiographic Memory for IVAs}

In light of the preceding review and discussion, we seek an approach to develop IVAs that, with a long-term autobiographic memory, capture in particular the social aspects of the agents' life stories in order to increase their believability from a user's perspective. People perceive and interpret the (social) world in terms of stories. Thus, we expect that users interacting with IVAs that possess, express, and act upon their life stories will be better able to interact with the agents and understand the nature of the agents' actions and stories that the IVAs try to express. Specifically, we expect that a user's degree of familiarity with an IVA correlates with his knowledge of its life stories.

In this section we start with the presentation of the memory design and certain criteria that are necessary to be considered regarding the memory structure and encoding mechanisms. Next we show the initial implementation and some samples of preliminary results. At the end we describe our evaluation plan for the validation of agents' life stories reconstructed from their long-term autobiographic memory.

\subsection{Design and On-going Implementation}

Before starting the technical design of a computational memory that allow IVAs to encode sensory data for their life story, a conceptual definition of life story is crucial for the task. Linde [11, page 21] stated that "A life story consists of all the stories and associated discourse units, such as explanations and chronicles and the connections between them, told by an individual during the course of his or her lifetime that satisfy the following two criteria:

1. The stories and associated discourse units contained the life story have as their primary evaluation a point about the speaker, not a general point about the way the world is.

2. The stories and associated discourse units have extended reportability; that is, they are tellable and are told and retold over the course of a long period of time ${ }^{2}$."

To adapt this definition and allow IVAs creating their believable and coherent life story, our design focuses on three essential story-associated discourse units: explanations, event chronology and evaluation; plus the characteristic of reportability.

Context and Agent Architecture The application which we use to test the IVAs' long-term autobiographic memory is called FearNot!. It is an antibullying software developed by partners in the eCIRCUS ${ }^{3}$ project. FearNot!

\footnotetext{
${ }^{2}$ Later in her book, Linde extended this definition by including the fact that the content of a life story, as a product of cultural context, to include certain facts and exclude others. Due to the space constraint in this paper, the cultural element in agents' life story is discussed in our on-going research work in [30].

${ }^{3}$ http://www.e-circus.org/
} 
provides eight-to-eleven year old users with the opportunity to visit a virtual school environment complete with characters representing most significant roles in bullying locations and scenarios that are commonplace in real-life bullying incidents. Users interact in the environment by watching narrative episodes unfolding as the result of the action executed by autonomous characters (the left screenshot in Figure 1). After each episode, the user can interact with one of the characters (the right screenshot in Figure 1), e.g. to give him/her suggestions on how to cope with the problem, and then see the result of the given advice on a subsequent episode.

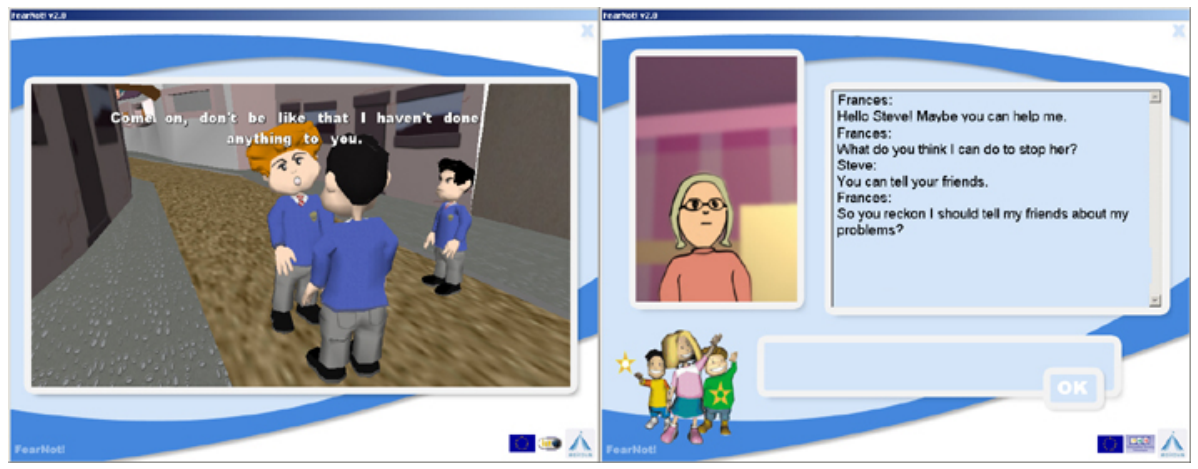

Fig. 1. Sample screenshots of FearNot! software: (Left) a bullying episode. (Right) user interaction interface.

The FAtiMA architecture (see details of implementation in [31]) was used as the foundation for integrating the long-term autobiographic memory. It is an agent architecture where emotions and personality take a central role in influencing behaviour. The concept of emotion used steams from OCC cognitive theory of emotions, where emotions are defined as valenced (good or bad) reactions to events. The assessment of this relationship between events and the character's emotions is called the appraisal process.

FAtiMA agents are able to perceive events/actions happening concurrently in the environment, e.g. the bystander character can perceive both events that the victim character is mocked by the bully while he/she is running away and crying. Nevertheless, agents record these events in a chronological sequence based on the time of the received messages from the framework. Moreover, to smooth the narrative storytelling and to achieve the educational goals of FearNot!, characters' goal is authored in a way that allows the storyline in each bullying episode to be consistent and easily understood by the child user who plays the game. Therefore, multiple agents may act within the same timeframe, but it is not our focus while developing FearNot! as a character-based narrative system. 
Memory Structure As inspired by the narrative structure for life story for humans, the storage structure representing each event in the computational autobiographic memory consists of three main components: Abstract, Narrative and Evaluation. To support the description of each component, Figure 2 shows an illustrative example of an event stored in one of the character's memories (the character is named John).

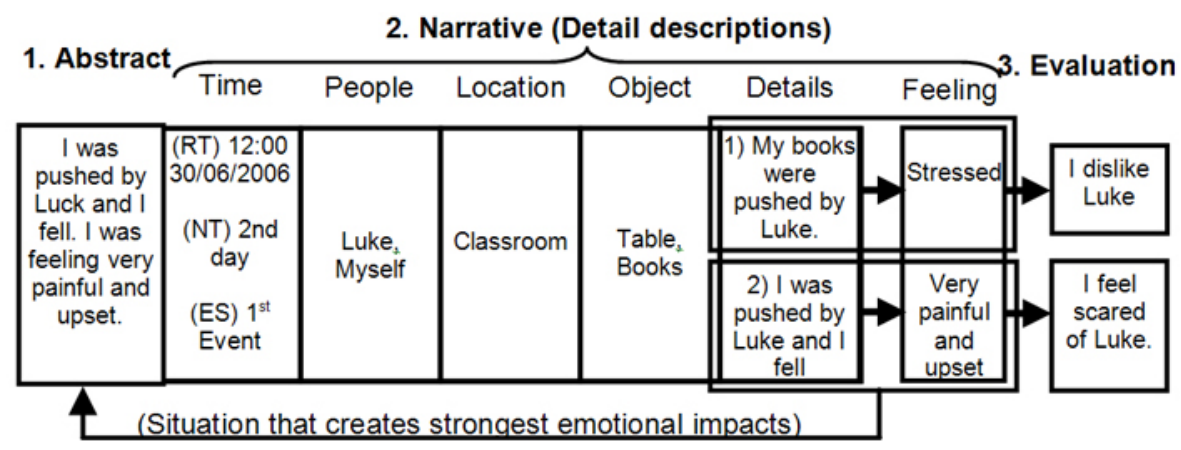

Fig. 2. A sample event constructed under an episode where a character (Luke) hits another one (John). The narrative component contains detailed descriptions of the event - when and where the event occurred, and objects and people (with their actions) involved in the event. The abstract of an event stores a summary of the event containing the most significant actions and feelings from the narrative component. Finally, evaluation corresponds to the agent's psychological evaluation of the effects of remembered actions according to the agent's social relations.

We aim to incorporate the important features extracted from Linde's definition of life story into the design of the memory storage structure. First, the chronological order of events is addressed by the inclusion of the Time in the autobiographic memory (see Figure 2). Recording the time and the chronological order of events is crucial for agents' life stories simply because users can make inferences about the causality and thereby meaning of events as experienced by the agent. The Time here records three different types of data:

- Real time (RT) is the time that represents the real world; it will be used for calculating the time difference when the software is used over a long term with a user.

- Narrative time (NT) indicates the virtual time when an episode takes place in the whole story. It will be particularly useful during the software evaluation session.

- Event sequence (ES) simply shows the order of the event in time, e.g. the first or second event.

Story explanation and evaluation are similar discourse units in agents' life stories. They refer to Abstract and Evaluation of an event in the memory 
structure respectively. Abstract is represented by the actions which, among others in the same event, were associated with the highest emotional intensities. The example in Figure 2 example shows that the Abstract contains the most significant action to John in the whole event: Being pushed by Luke and falling which caused him to feel very bad. In agents' life stories, Abstract serves as an interactive function which often summarises the narrative or provides a "hint" to reveal the rest of the story (i.e. details stored in Narrative). The explanation is thus embedded in the design - in a human life story, it starts with a statement of some proposition, and then follows it with a sequence of statements of reasons showing why the proposition should be believed [11].

Based on the context of FearNot - an educational anti-bullying software, an event's Evaluation indicates the effects of remembered actions according to the agent's social relations, i.e., this essentially shows the action's consequence(s) in terms of inter-personal relationships remembered in an agent's memory. This evaluation in the agents' life stories is socially and interactionally important since it shows their "personalised" view, not a general point about the way the world is. It also plays a critical role in establishing the coherence of a life story, because an agent can trace back to many similar past experiences, from which the same result of evaluation was derived. Retrieving these experiences from autobiographic memory and putting them together in the agent's life story reconstructs the kind of "self" that is presented by the agent.

While retrieving events from agent's autobiographic memory, depending on the context, a set of search keys is used to look for similar events. For examples, the victim character can search for all past experiences associated with a specific person (e.g. the bully), happened in the playground, and with negative emotions. Here the content manipulations, which group similar past events together and then select representative contents for story generation, are similar to Event Reconstruction and Event Filtering and Ranking processes respectively from our previous research [29]. Furthermore, we expect that it would be more believable to have some random factors influencing the process of story creation, like humans telling their life story with slightly random content, structure and length. Therefore, an algorithm for constructing the remembered stories randomly is applied here to pick-up contents from some Narrative fields (see Figure 2). Nevertheless, being consistent with the basic structure of a narrative is very important since we want to retain the meaning of each story from an individual agent's perspective.

Last but not least, the characteristic of reportability is shown in the way that actions are remembered in an agent's autobiographic memory: The significance of an action to the agent is determined by the emotion attributed to that action from the appraisal process ${ }^{4}$. To be reportable an event/action must be unusual,

\footnotetext{
${ }^{4}$ In the current implementation of FAtiMA, when an external event or action is perceived, a set of predefined emotional reaction rules is used by an individual agent in the appraisal process to create the meaning of the event. These rules, which include emotional threshold, importance of goals, desirability of events, etc., represent the agent's standards and attitudes towards generic events.
} 
unexpected, or abnormal; neutral actions/events that did not create much emotional impact to the agent are probably not "worth telling" [32] and will not be remembered as part of its life story. In our previous work an event reconstruction process in the agent's memory retrieval can generate short summaries covering the most significant actions in an event, derived from the autobiographic memory [33]. Thus, the agent can respond with narratives when interacting with users. We expect that similar mechanisms can be applied to create an agent's life story which contains events taking place under a specific context in different periods of the agent's life time. Therefore, stories generated from the event reconstruction process are context-dependent - the context thus serves as a matching key for selecting relevant events for the event reconstruction.

\subsection{Preliminary Results}

Based on our previous work [33] which incorporated the psychological view that emotions can arise in response to both internal and external events, agents embedded with long-term autobiographic memory are now able to encode events that happen during their life time. In the same context of using FearNot! software, Figure 3 illustrates, in XML format, part of the memory contents that an agent encoded in its memory.

The implementation is still on-going at the current stage. Both Abstract and Evaluation will be created in each event as planned in the design. A language engine will be used to convert the memory contents into agent's life story. Examples of an agent's event summary generated from our previous work [33] are as follows:

- "During this morning. Luke made fun of me on the street and then Luke continued teasing me. I was sad. Can you believe it. I considered to fight Luke back and then I was planning to stay safe. I was feeling scared. Then, I failed to fight Luke back."

- "This morning, I looked at Luke on the street and I hate Luke really, Luke attacked me and then I cried. Did you see that, Luke told me to get out of the way. I was feeling very desperate, I asked Luke to stop bullying me."

\subsection{Evaluation Plan}

Undoubtedly it would be inappropriate to compare real life stories from humans with our agents' synthetic ones, because a human life story is extremely dynamic, discontinuous and with rich social discourse units that conveys "who we are and how we got to be that way". Nevertheless, in our future evaluation with eightto-eleven year old child users, we eagerly aim to examine the story structure and the level of coherence of life stories which will be generated from the long-term autobiographic memory. Through questionnaire and story-rewriting exercises, we hope to find out respectively 1) what length, quality and contents of the life story users would expect from our agents; and 2) how similar the agents' life story can be to users' written summary based on their observation on the narrative 


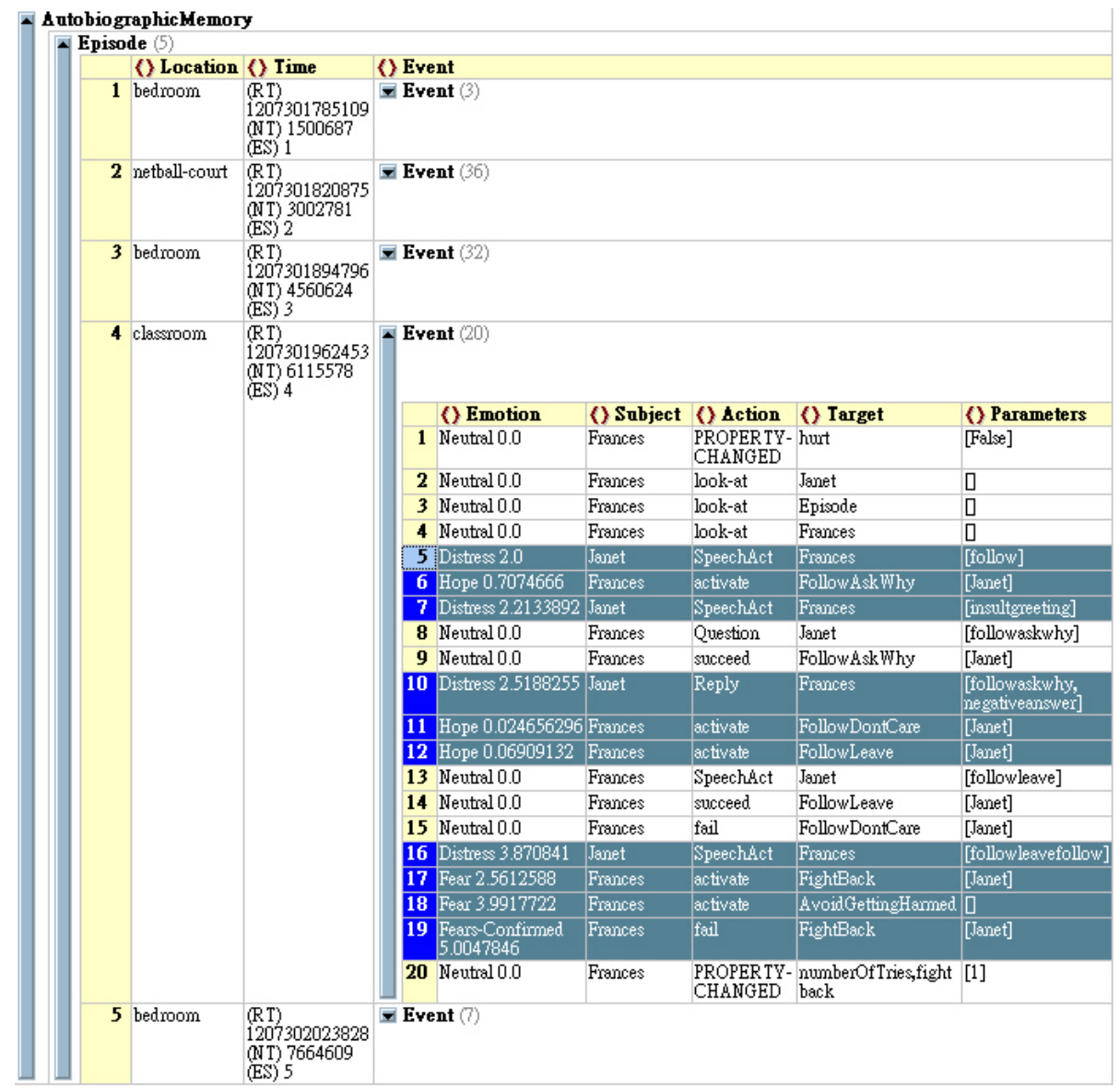

Fig. 3. Contents of an agent (a female victim character in FearNot!) showing Frances' long-term autobiographic memory in a life story structure. The highlighted part shows significant actions that created different types of emotional impact to the agent in the event. The view in this figure was generated by Altova XMLSpyß). 
unfolded in FearNot!. To answer these questions, we have recently carried out an evaluation event including school children interacting with several FearNot! episodes, using an initial implementation of the architecture described in this article. We are currently analysing the results that will be presented in future publications.

Ultimately, in future user studies supported by the fully implemented architecture, we expect to find out whether the long-term believability of narrative agents can be increased through the integrated autobiographic memory.

\section{Conclusion and Future Work}

In this paper we discussed the issue of long-term believability for IVAs. We addressed this issue by proposing to integrate a long-term autobiographic memory for the creation of agents' coherent life stories as their narrative "self". We took inspiration from human life stories and illustrated an on-going implementation for agents' storytelling memory structure, which essentially incorporates human narrative discourse units and the reconstructive nature of human autobiographic memory. Finally, through the creation of their own life stories, we expect that this memory can help agents to 1) express their internal subjective sense of "self" and 2) show continuity of the "self" - autobiographic agents that learn and adapt themselves in the environment.

In the future we will first focus on finishing the architecture implementation and then establish the link between the long-term autobiographic memory and a language engine ${ }^{5}$ for making agents' life story readable to human users. Furthermore, we will investigate making an agent's story more recognisable as an interesting life story, particularly taking into account that the coherence is a cooperative achievement of the 'story-teller' (the agent) and the 'audience' (the user). This indicates that agents must construct each narrative with an appropriate evaluation, plus giving the audience sufficient cues to understand this evaluation.

\section{Acknowledgments}

Authors would like thank members of the eCIRCUS technical team to create the FearNot! software framework as a testbed for the work described in this paper, and particularly João Dias from INESC-ID for developing the FAtiMA architecture. This work was partially supported by European Community (EC) and is currently funded by the eCIRCUS project IST-4-027656-STP. The authors are solely responsible for the content of this publication. It does not represent the opinion of the EC, and the EC is not responsible for any use that might be made of data appearing therein.

\footnotetext{
${ }^{5}$ In the previous implementation FAtiMA sends the contents of a character's short summary generated from autobiographic memory to the language engine as speech acts. The language engine then makes use of the semantic parser for the generation of natural language used in the summary. See [34] for detail.
} 


\section{References}

[1] Bates, J.: The role of emotion in believable agents. Communication of the ACM 37(7) (1994) 122-125

[2] Bratman, M.E.: Intention, Plans, and Practical Reason. Harvard University Press, Cambridge, MA (1987)

[3] Ortony, A., Clore, G., A., C.: The cognitive structure of emotions. Cambridge University Press, Cambridge, UK (1988)

[4] Dörner, D.: The mathematics of emotions. In Dörner, D., Detje, F.and Schaub, H., eds.: Proceedings of the Fifth International Conference on Cognitive Modeling, Bamberg, Germany (April, 10-12 2003) 75-79

[5] Dörner, D., Gerdes, J., Mayer, M., Misra, S.: A simulation of cognitive and emotional effects of overcrowding. In: Proceedings of the 7 th International Conference on Cognitive Modeling, Trieste, Italy (Apr, 5-8 2006)

[6] Barthes, R.: Introduction to the structural analysis of narrative. In: Image Music Text (1977). New York: Hill and Wang (1966) 79-124

[7] Goguen, J.: The structure of narrative. URL http://wwwcse.ucsd.edu/classes/sp01/cse171/story.html (2001)

[8] Aylett, R.: Narrative in virtual environments: Towards emergent narrative. In: Proc. Narrative Intelligence. AAAI Fall Symposium 1999, AAAI Press (1999) 83-86 Technical Report FS-99-01.

[9] Aylett, R., Figueiredo, R., Louchart, S., Dias, J., Paiva, A.: Making it up as you go along: improvising stories for pedagogical purposes. In: Intelligent Virtual Agents Conference (IVA). Volume 4133., Springer Berlin / Heidelberg, Springer LNAI (2006) 307-315

[10] Sengers, P.: Narrative and schizophrenia in artificial agents. In Mateas, M., Sengers, P., eds.: Narrative Intelligence. Amsterdam: John Benjamins (2003) 259-278

[11] Linde, C.: Life Stories: The Creation of Coherence. Oxford University Press (1993)

[12] Abbott, H.P.: The cambridge introduction to narrative. Cambridge University Press, Cambridge (2002)

[13] Schank, R.C., Abelson, R.P.: Scripts, Plans, Goals and Understanding: An Inquiry into Human Knowledge Structures. Lawrence Erlbaum Assicuates, Hillsdale, New Jersey (1977)

[14] Minsky, M.: A framework for representing knowledge. In Winston, P.H., ed.: The Psychology of Computer Vision. New York: McGraw-Hill (1975) 211-277

[15] Endo, Y.: Anticipatory robot control for a partially observable environment using episodic memories. Technical Report GIT-IC-07-03, Georgia Tech Mobile Robot Lab (2007)

[16] Ogino, M., Ooide, T., Watanabe, A., Asada, M.: Acquiring peekaboo communication: Early communication model based on reward prediction. In: Proceedings of IEEE International Conference in Development and Learning (ICDL) 2007, London, UK (July 2007)

[17] Mirza, N.A., Nehaniv, C.L., Dautenhahn, D., te Boekhorst, R.: Interaction histories: From experience to action and back again. In: Proceedings of the 5th IEEE International Conference on Development and Learning (ICDL 2006), Bloomington, IN, USA (2006) ISBN 0-9786456-0-X.

[18] Mirza, N.A., Nehaniv, C.L., Dautenhahn, D., te Boekhorst, R.: Grounded sensorimotor interaction histories in an information theoretic metric space for robot ontogeny. Adaptive Behavior 15(2) (2007) 167-187 
[19] Nuxoll, A., Laird, J.E.: A cognitive model of episodic memory integrated with a general cognitive architecture. In: Proceedings of the Sixth International Conference on Cognitive Modeling, Mahwah, NJ, Lawrence Earlbaum (2004) 220-225

[20] Tecuci, D., Porter, B.: A generic memory module for events. In: Proceedings to the 20th Florida Artificial Intelligence Research Society Conference (FLAIRS 20), Key West, FL (2007)

[21] Brom, C., Pesková, K., Lukavskýz, J.: What does your actor remember? towards characters with a full episodic memory. In Cavazza, M., Donikian, S., eds.: International Conference on Virtual Storytelling. Volume 4871 of Lecture Notes in Computer Science., Springer (2007) 89-101

[22] Bruner, J.: The narrative construction of reality. Critical Inquiry 18(1) (1991) $1-21$

[23] Schank, R.C., Abelson, R.P.: Knowledge and memory: The real story. In Wyer, R.S., ed.: Knowledge and Memory: The Real Story. Lawrence Erlbaum Associates (1995) 1-85

[24] Dautenhahn, K.: Story-telling in virtual environments. In: Working Notes Intelligent Virtual Environments, Workshop at the 13th biannual European Conference on Artificial Intelligence (ECAI-98), Brighton Centre, Brighton, UK (23-28 August 1998)

[25] Dautenhahn, K.: Embodiment in animals and artifacts. In: AAAI FS Embodied Cognition and Action, AAAI Press (1996) 27-32 Technical report FS-96-02.

[26] Nelson, K.: The psychological and social origins of autobiographical memory. Psychological Science 4 (1993) 7-14

[27] Dautenhahn, K., Coles, S.: Narrative intelligence from the bottom up: A computational framework for the study of story-telling in autonomous agents. Artificial Societies and Social Simulation (2000) 31st January 2001.

[28] Ho, W.C., Dautenhahn, K., Nehaniv, C.L.: A study of episodic memory-based learning and narrative structure for autobiographic agents. In: Proceedings of Adaptation in Artificial and Biological Systems, AISB 2006 conference. Volume 3. (2006) 26-29

[29] Ho, W., Dautenhahn, K., Nehaniv, C.L.: Computational memory architectures for autobiographic agents interacting in a complex virtual environment: A working model. Connection Science 20:1 (2008) $21-65$

[30] Ho, W.C., Dautenhahn, K., Lim, M., Enz, S., Zoll, C., Watson, S.: Towards learning 'self' and emotional knowledge in social and cultural human-agent interactions. International Journal of Agent Technologies and Systems 1 (2009) To appear

[31] Dias, J., Paiva, A.: Feeling and reasoning: a computational model for emotional agents. In: In Proceedings of 12 th Portuguese Conference on Artificial Intelligence (EPIA 2005), Springer (2005) 127-140

[32] Bruner, J.: Actual Minds, Possible Worlds. Harvard University Press, Cambridge, MA (1986)

[33] Ho, W.C., Dias, J., Figueiredo, R., Paiva, A.: Agents that remember can tell stories: Integrating autobiographic memory into emotional agents. In: Autonomous Agents and Multiagent Systems (AAMAS), ACM Press (2007) 35-37

[34] Dias, J., Ho, W.C., Vogt, T., Beeckman, N., Paiva, A., Andre, E.: I know what i did last summer: Autobiographic memory in synthetic characters. In: Affective Computing and Intelligent Interaction. Volume 4738/2007 of Lecture Notes in Computer Science., Springer Berlin/Heidelberg (2007) 606-617 